\title{
Predator: A Practical Tool for Checking Manipulation of Dynamic Data Structures Using Separation Logic ${ }^{\star}$
}

\author{
Kamil Dudka, Petr Peringer, and Tomáš Vojnar \\ FIT, Brno University of Technology, Czech Republic
}

\begin{abstract}
Predator is a new open source tool for verification of sequential C programs with dynamic linked data structures. The tool is based on separation logic with inductive predicates although it uses a graph description of heaps. Predator currently handles various forms of lists, including singly-linked as well as doubly-linked lists that may be circular, hierarchically nested and that may have various additional pointer links. Predator is implemented as a gCC plug-in and it is capable of handling lists in the form they appear in real system code, especially the Linux kernel, including a limited support of pointer arithmetic. Collaboration on further development of Predator is welcome.
\end{abstract}

\section{Introduction}

In this paper, we present a new tool called Predator for fully automatic verification of sequential C programs with dynamic linked data structures. In particular, Predator can currently handle various complex kinds of singly-linked as well as doubly-linked lists that may be circular, shared, hierarchically nested, and that can have various additional pointers (head/tail pointers, data pointers, etc.). Predator implicitly checks for absence of generic errors, such as null dereferences, double deletion, memory leakage, etc. It can also print out a symbolic representation of the shapes of the memory structures arising in a program. Finally, users can, of course, use Predator to check custom properties about the data structures being used in their code by writing (directly in C) tester programs exercising these structures.

Predator is based on separation logic with higher-order inductive predicates. It is inspired by the works [2 9]10] and the very influential tool called Space Invader (or simply Invader). However, compared to Invader, the heap representation in Predator is not based on lists of separation logic formulae, but rather a graph representation of these formulae. The algorithms handling the symbolic heap representation (in particular, the abstraction and join operators based on detecting occurrences of heap structures that can be described by inductive predicates) have been newly designed.

Compared to Invader that contains a partial support of doubly-linked lists only, Predator supports them equally well as singly-linked lists. Predator also contains a special support for list segments of length 0 or 1 that are common in practice [9] and that may cause problems to Invader (as we illustrate further on).

\footnotetext{
* This work was supported by the Czech Science Foundation (project P103/10/0306), the Czech Ministry of Education (projects COST OC10009 and MSM 0021630528), and the BUT FIT project FIT-S-11-1. An extended version of the paper is available as the technical report [6].

1 http: / / www . eastlondonmassive.org/East_London_Massive/Invader_Home.html

G. Gopalakrishnan and S. Qadeer (Eds.): CAV 2011, LNCS 6806, pp. 372-378, 2011.

(C) Springer-Verlag Berlin Heidelberg 2011
} 
The long term goal of Predator is handling real system code, in particular, the Linux kernel. In such code, for efficiency reasons, special forms of lists are used. In order to be able to handle them, Predator comes with a limited support of pointer arithmetic, which, however, covers most practical needs. Therefore, in a heap representation, the points-to links are associated with an offset w.r.t. the object they point to. Despite such an extension is not mentioned in [210], the Invader tool seems to partially support it, but it fails in many practical cases that Predator can handle well.

Predator is written in $\mathrm{C}++$. It is built as a gCC plug-in, hence its front end is the same compiler that is used in practice for compiling the code that Predator is intended to analyse. Predator is completely open source 2 in order to allow for an open collaboration on its further development, which is very welcome.

There of course exist many other works on verification of programs with dynamic linked data structures than those using separation logic, including works based on other logics [7], automata [3], upward-closed sets [1], etc. These approaches offer different degrees of generality, automation, or scalability. A proper discussion of such works is, however, beyond the scope of this tool paper. Throughout the paper, we instead concentrate on a comparison with Invader as the closest tool to Predator. A similar tool is also jStar [5] which, however, concentrates on Java-specific problems. In [4], a bi-abductive analysis based on separation logic was proposed and implemented in a version of Invader, called Abductor 3 . This analysis, which is more scalable but less precise than the classical analysis used in Invader and Predator, is not yet implemented in Predator (whose core can, however, be used to implement it in the future). Unlike Invader, Predator cannot currently handle entire modules of Linux (such as drivers) due to a so far very weak support of non-pointer data, which is one of the planned future works on Predator (together with a support of tree data structures, bi-abduction, etc.).

Below, we first say a bit more on the Linux lists supported by Predator, then we briefly mention some implementation details of Predator, and we proceed to interesting cases studies that illustrate the power of our tool. For some of the case studies, we are not aware of any other fully automatic, freely available tool, capable of handling them.

\section{Lists Used in The Linux Kernel}

As there is no standard implementation of linked lists in the $\mathrm{C}$ language, the Linux kernel has to implement lists on its own. The list implementation in Linux is wellknown for its efficiency, portability, readability, and scalability-for instance, it allows to create list nodes which are owned by many distinct lists at a time. The downside is that it operates at a low level, hence it is easy to misuse the routines, and cause a disaster within the kernel. Later on, we will mention some common mistakes in manipulating Linux lists, which Predator is able to detect.

\footnotetext{
${ }^{2}$ http://www.fit.vutbr.cz/research/groups/verifit/tools/predator/

${ }^{3}$ Abductor is publicly available, but we have not managed to make it run. There is also a commercial implementation (www. monoidics.com), which is, however, not freely available.

${ }^{4}$ Although Invader has already shown some interesting results on pre-processed source code of selected Linux drivers, it is not ready for analysing drivers using the native Linux lists.
} 


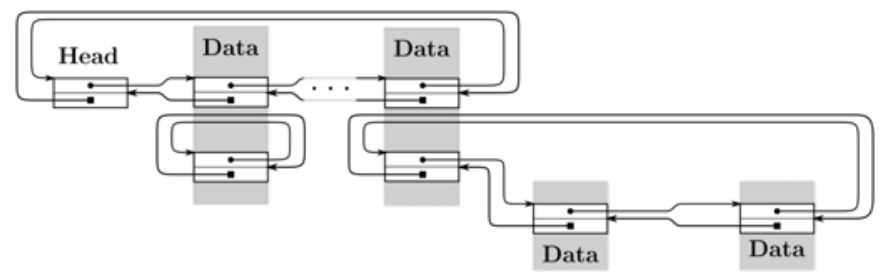

Fig. 1. A list of lists as implemented in the Linux kernel

The whole implementation of Linux lists is available in a single header file and consists of about 500 lines of code. It defines only one 5 type, which does all the job. The type contains a pair of pointers (next and prev), but no data. Such a structure is called a head and can be used in two ways - either as a starting point of a list (a standalone head), cf. the leftmost node in Fig. 1. or as part of list nodes (an embedded head).

Basic list operations (like addition, removal, reconnection of nodes) work only with heads and do not care about any associated data. In particular, the routines themselves do not distinguish between embedded and standalone heads.

The embedded head can be placed at an arbitrary offset in the surrounding structure. Moreover, it is possible to put many embedded heads into one structure such that one node is part of many lists. The standalone head can be placed on stack, but it can also be surrounded by another type. This way one can construct hierarchical list structures as shown in Fig. 1. Note that the beginning of the data nodes (depicted in gray in Fig.1) needs not to be directly accessible by any pointer and can hence be mistakenly considered garbage if pointer arithmetic is not taken into account.

Linux lists are doubly-linked and circular, which significantly simplifies the design and boosts performance. That is, each routine for reconnection of a node (insertion, deletion, etc.) fits into a single basic block, which would not have been possible in case of regular NULL-terminated doubly-linked lists. It also implies that there is no need to have an explicit starting point (a standalone head) for each list. The Linux list library provides macros to define a standalone head and initialise it as an empty list. In case of Linux lists, an empty list means that next and prev fields point to the head itself.

The basic list traversal macro (list_for_each) provides a pointer to a head in each iteration and works without any type-awareness of the data nodes being traversed. The macro list_entry then allows to translate a pointer to a head into a pointer to the corresponding data node. As the offset of each embedded head in the structure is known at compile-time, the macro can easily use pointer arithmetic to compute the required address. There is also an extended macro for list traversal (list_for_each_entry) that efficiently wraps list_entry and this way gives us a pointer to the data node in each iteration, instead of the pointer to the embedded head. Note, however, that it may happen that some pointer variable points to the unallocated space around a standalone head and yet may be correctly used (by being subsequently moved forward by pointer arithmetic). On the other hand, dereferencing such a pointer is an error, which can, e.g., lead to stack smashing (and is detectable by Predator).

\footnotetext{
${ }^{5}$ Starting with Linux-2.5.64, there is also an optimised variant of lists for constructing hash tables. We are not yet able to analyse the code that uses these optimised lists.
} 
A nice introduction into how Linux lists work can be found in [8]. We use the code from there as one of the case studies distributed as test-cases with Predator.

\section{A Note on the Implementation of Predator}

Predator implements a symbolic analysis based on separation logic with higher-order inductive predicates, inspired by the works [210] implemented in Space Invader. Predator uses a graph representation of separation logic formulae, a little bit similar to the graph representation introduced in [2] for description of the predicate discovery algorithm. Our representation is, however, more complex and used all the time.

In our graph-based symbolic representation of heaps, we use two kinds of nodes: $o b$ jects (statically and automatically allocated program variables, dynamically allocated storage, etc.) and values of the objects (e.g., addresses of objects and the special undefined, deleted, and null values in the case of pointers and function pointers). Objects can be nested in order to represent the composition of $\mathrm{C}$ language structures. The appropriate nodes are linked by oriented graph edges hasValue (going from objects to values) and pointsTo (going from values to target objects). In order to allow for efficient equality testing, equal objects are simply linked to the same value node. To encode non-equality relations, value nodes may be linked by undirected neq edges. Further, when pointer arithmetic produces a value that does not point to a valid target, we use so-called offset edges between value nodes. Such values can later be used for a valid memory operation - they can either be translated by another use of pointer arithmetic to a valid address, or directly used for accessing an existing subobject of a non-existent surrounding object (which is common, e.g., when working with Linux lists).

We represent inductive predicates as special abstract objects. Currently, we support only singly- and doubly-linked list segments that may be shared, nested, and with various additional (head, tail, data, and the like) pointers. A doubly-linked list segment (DLS) has two endpoints, both of which may be pointed to. Therefore, since each object has exactly one address, we in fact represent DLS as pairs of abstract objects. To cope with pointer arithmetic, we equip abstract objects with offsets specifying the relative placement of the core linking pointers (next/prev for lists). Moreover, to cope with Linux lists, we also record the head offset that is a relative placement of the list pointer's target. For Linux lists, it corresponds to the offset of the embedded head, whereas for regular lists, it is simply zero. We do not treat the minimal segment length as an explicit property of a list segment as in [9]. Instead, our list segments are implicitly possibly empty (i.e., of length $0+$ ). We use the generic mechanism of neq edges between nodes before and after a segment to construct non-empty segments (length 1+). For DLS, we use two neq edges for length 1+ (one edge for each direction) and three neq edges for length $2+$ (the additional neq edge is in between the ends of the DLS). Such an approach leads to a simpler and more readable implementation. Apart from that, we then have special abstract objects for list segments of length 0 or 1 .

Predator maintains a set of symbolic heaps for each basic block entry. The set is not yet implemented as an ordered or hashed container, but it utilises a join operator similar 
to the join operator introduced in [10], helping to significantly decrease the number of symbolic heaps to be maintained. Moreover, Predator uses a slightly modified version of the join algorithm to merge pairs of objects during a list segment abstraction, in particular to join nested predicates, shared (head, tail or other) pointers, and other data. The modified join algorithm operates on two parts of a single heap given by the pair of objects being merged, and constructs a joint description of both parts. The algorithm can also run in a read-only mode to decide whether the join operation is possible. The readonly mode can be safely used during predicate discovery. Thanks to this, the algorithms for abstraction and predicate discovery are implemented as a very thin purpose-specific layer on top of the generic join algorithm.

For inter-procedural analysis, Predator uses function summaries in a way similar to [10], including a support of indirect function calls and recursive calls of fixed depth.

Predator is tightly integrated with $\mathrm{gcc}$ (version 4.5.0 and newer) as a plug-in. Therefore, there is no need to manually pre-process the sources, neither to change the way they are built, whenever dealing with software natively compiled by gcc. Usage of Predator is as easy as adding a new compiler flag into CFLAGS while building a project. Code defects encountered during analysis are reported in the $\mathrm{gCC}$ format. Hence it is easy to reuse existing development tools, IDE, etc. In order to give users a clue about detected errors, Predator provides a backtrace for each error. Predator attempts to report as many errors and warnings as possible per run. For instance, if a memory leak is detected, a warning is issued, and Predator keeps searching for further errors (due to a garbage collector that gets the symbolic heap back to its consistent shape). Predator supports error recovery for most of the program errors which it is able to detect.

\section{Experiments with Predator}

Along with Predator, we distribute a comprehensive set of programs (over a hundred test cases) that can be handled by our tool, including various textbook implementations of lists (singly-linked, doubly-linked, circular, hierarchically nested, etc.) as well as examples using Linux list 6. These case studies are mid-size (up to 300 lines), however, they contain almost only pointer manipulations unlike larger programs whose big portions are often not relevant for pointer analyses like ours. Apart from basic list manipulation (creation of random lists, reversal, destruction, etc.), we provide also examples of various sorting algorithms: Merge-Sort, Insert-Sort, and Bubble-Sort7. The Merge-Sort case study operates on hierarchical singly-linked lists. The other two sorts use the native implementation of Linux lists. Predator is not proving that the resulting list is sorted, but it verifies memory safety of the code. Invader, as a freely available tool closest to Predator, is not able to analyse any of our sorting case studies.

Some of our test cases show common mistakes in using Linux lists such as mixing pointers to a head with pointers to data or treating a standalone head as if it was an embedded head. Only programmers know the purpose of each head, and if they use the

\footnotetext{
${ }^{6}$ https://github.com/kdudka/predator/tree/61d5df3/sl/data

${ }^{7}$ See [6] for file names under which the case studies can be found in the distribution of Predator.
} 
head in a wrong way, it is likely to be noticed at run-time only (and often not immediately). For example, starting from a standalone head, the list_for_each_entry macro provides a valid pointer to data in each iteration. However, if one starts to traverse the list from the middle, it ends up by misinterpreting the standalone head's neighbourhood as list node's data. Predator is capable of detecting such mistakes. We, for instance, provide an example where a wrong head is used for a Linux list traversal. Despite even the dynamic analysis tool valgrind, often used by developers, claims there is an invalid write, Invader says the code is safe. On the contrary, Predator detects the flaw in $0.01 \mathrm{~s}$, which is even faster than valgrind.

Our test suite further contains various programs intended to stress test the discovery of inductive predicates. These case studies include, e.g., conversion of a singlylinked list into a doubly-linked and then back to singly-linked list, or construction of two independent lists starting from the same node, which other tools may inaccurately over-approximate as a hierarchically nested list or a binary tree.

Another case study considers a call of free ( ) on an embedded head that appears in real code if the head is placed at zero offset within the data node. Tools that ignore address aliasing of fields placed at the same offset, like Invader, mistakenly report such an operation as an error in the analysed program. Since Predator uses the offset-based description of list segments, it can easily cope with address aliasing.

We also provide a few case studies of lists where each node optionally owns some nested objects. Those may be incorrectly abstracted as nested lists if only usual list segments are considered, and in case the program does not really treat such objects as lists, it leads to spurious memory leaks or even non-termination of the analysis. Predator covers these cases by special abstract objects of length 0 or 1 , which allows a more precise analysis and solves the problems with spurious errors and non-termination.

Across all our case studies, Predator acts fully automatically. There is no need to tell Predator what kind of data structures to look for. Given a C program, it simply returns the corresponding list of errors and warnings. In all but one of the mentioned tests, the time consumption was under 1.0s on Intel Core i5 3.33GHz. Moreover, for a vast majority of the tests, it was under $0.1 \mathrm{~s}$. The only exception was the Merge-Sort example, which took 7.8 s to analyse. We are, however, not aware of any comparable tool that is able to analyse the same example faster.

\section{Conclusion}

We have presented Predator, a new separation logic based tool for analysing programs with dynamic linked data structures. Despite the tool is only at the beginning of its development, we have argued that it already offers many interesting features. In the future, the tool should be, e.g., enriched with some (preferably light-weight) support of non-pointer data (integers, arrays), extended to handle further classes of dynamic data structures, extended to handle $\mathrm{C}++$ code (which the gcc-based front-end can easily handle), and so on. Since Predator is open source, GPL-licensed, and written such that its code is readable, collaboration on its further development is very well possible. 


\section{References}

1. Abdulla, P.A., Bouajjani, A., Cederberg, J., Haziza, F., Rezine, A.: Monotonic Abstraction for Programs with Dynamic Memory Heaps. In: Gupta, A., Malik, S. (eds.) CAV 2008. LNCS, vol. 5123, pp. 341-354. Springer, Heidelberg (2008)

2. Berdine, J., Calcagno, C., Cook, B., Distefano, D., O'Hearn, P.W., Wies, T., Yang, H.: Shape Analysis for Composite Data Structures. In: Damm, W., Hermanns, H. (eds.) CAV 2007. LNCS, vol. 4590, pp. 178-192. Springer, Heidelberg (2007)

3. Bouajjani, A., Habermehl, P., Rogalewicz, A., Vojnar, T.: Abstract Regular Tree Model Checking of Complex Dynamic Data Structures. In: Yi, K. (ed.) SAS 2006. LNCS, vol. 4134, pp. 52-70. Springer, Heidelberg (2006)

4. Calcagno, C., Distefano, D., O'Hearn, P.W., Yang, H.: Compositional Shape Analysis by Means of Bi-abduction. In: Proc. of POPL 2009. ACM Press, New York (2009)

5. Distefano, D., Parkinson, M.: jStar: Towards Practical Verification for Java. In: Proc. of OOPSLA 2008, ACM Press, New York (2008)

6. Dudka, K., Peringer, P., Vojnar, T.: Predator: A Practical Tool for Checking Manipulation of Dynamic Data Structures Using Sep. Logic. Tech. rep. FIT-TR-2011-02, FIT BUT (2011)

7. Sagiv, S., Reps, T., Wilhelm, R.: Parametric Shape Analysis via 3-valued Logic. TOPLAS 24(3) (2002)

8. Shanmugasundaram, K.: Linux Kernel Linked List Explained (2005), http://isis.poly.edu/kulesh/stuff/src/klist

9. Yang, H., Lee, O., Calcagno, C., Distefano, D., O’Hearn, P.W.: On Scalable Shape Analysis. Technical report RR-07-10, Queen Mary, University of London (2007)

10. Yang, H., Lee, O., Berdine, J., Calcagno, C., Cook, B., Distefano, D., O’Hearn, P.W.: Scalable Shape Analysis for Systems Code. In: Gupta, A., Malik, S. (eds.) CAV 2008. LNCS, vol. 5123, pp. 385-398. Springer, Heidelberg (2008) 